OPEN ACCESS

Edited by:

Dimos Baltas,

University of Freiburg Medical Center,

Germany

Reviewed by:

Francesco Ricchetti,

Sacro Cuore Don Calabria Hospital

(IRCCS), Italy

Nikolaos Tselis,

University Hospital Frankfurt, Germany

${ }^{*}$ Correspondence:

Jun Itami

jitami@ncc.go.jp

Specialty section:

This article was submitted to

Radiation Oncology,

a section of the journal

Frontiers in Oncology

Received: 05 November 2021 Accepted: 16 December 2021 Published: 13 January 2022

Citation:

Itami J, Murakami N, Watanabe M,

Sekii S, Kasamatsu T, Kato $S$, Hirowatari H, Ikushima H, Ando K, Ohno T, Okamoto $H$, Okuma $K$ and Igaki H (2022) Combined Interstitial and Intracavitary High-Dose Rate Brachytherapy of Cervical Cancer.

Front. Oncol. 11:809825

doi: 10.3389/fonc.2021.809825

\section{Combined Interstitial and Intracavitary High-Dose Rate Brachytherapy of Cervical Cancer}

\author{
Jun Itami ${ }^{1,2 *}$, Naoya Murakami ${ }^{2}$, Miho Watanabe $^{3}$, Shuhei Sekii ${ }^{4}$, Takahiro Kasamatsu ${ }^{5}$, \\ Shingo Kato ${ }^{6}$, Hisako Hirowatari ${ }^{7}$, Hitoshi Ikushima ${ }^{8}$, Ken Ando ${ }^{9}$, Tatsuya Ohno ${ }^{10}$, \\ Hiroyuki Okamoto ${ }^{11}$, Kae Okuma ${ }^{2}$ and Hiroshi Igaki ${ }^{2}$ \\ ${ }^{1}$ Shin-Matsudo Accuracy Radiation Therapy Center, Shin-Matsudo Central General Hospital, Chiba, Japan, ${ }^{2}$ Department of \\ Radiation Oncology, National Cancer Center Hospital, Tokyo, Japan, ${ }^{3}$ Department of Radiology, Chiba University Graduate \\ School of Medicine, Chiba, Japan, ${ }^{4}$ Department of Radiation Oncology, Hyogo Prefectural Cancer Center, Hyogo, Japan, \\ ${ }^{5}$ Department of Gynecology, Tokyo Metropolitan Bokuto Hospital, Tokyo, Japan, ${ }^{6}$ Department of Radiation Oncology, \\ International Medical Center, Saitama Medical University, Saitama, Japan, ${ }^{7}$ Department of Radiation Oncology, Tokyo Rinkai \\ Hospital, Tokyo, Japan, ${ }^{8}$ Department of Radiation Oncology, Tokushima University Faculty of Medicine, Tokushima, Japan, \\ ${ }^{9}$ Department of Radiation Oncology, Gunma Cancer Center, Gunma, Japan, ${ }^{10}$ Department of Radiation Oncology, Gunma \\ University Graduate School of Medicine, Gunma, Japan, ${ }^{11}$ Department of Medical Physics, National Cancer Center Hospital, \\ Tokyo, Japan
}

High-dose-rate brachytherapy by remote afterloading is now performed under threedimensional image guidance by CT or MRI. Three-dimensional image-guided brachytherapy in cervical cancer disclosed that the traditional intracavitary brachytherapy by Manchester method cannot deliver an adequate dose to the large tumor with resulting local recurrence. To improve the local control rate, combined interstitial and intracavitary (hybrid) brachytherapy can increase the dose to the large parametrial involvement without increasing the dose to the rectum and bladder. Whether hybrid brachytherapy can be performed safely on a multi-institutional basis remains to be studied. From 2015, phase I/II study of hybrid brachytherapy was launched in Japan, and it was revealed that hybrid brachytherapy can be performed safely and with a high quality of radiation dose distribution in a multi-institutional study. In Japan, the number of patients undergoing hybrid brachytherapy in cervical cancer is rapidly rising. Education and clinical trial are very important to establish hybrid brachytherapy in the management of cervical cancer.

Keywords: cervical cancer, interstitial, intracavitary, brachytherapy, three-dimensional image-guided

\section{IMAGE-GUIDED BRACHYTHERAPY OF THE CERVICAL CANCER}

Brachytherapy has been applied mainly in head and neck cancers, cutaneous cancers, gynecological cancers, and prostate cancers. Currently, most brachytherapy is performed in a high-dose rate (HDR) because HDR brachytherapy does not require a radiation-shielded ward and enables image-guided optimization easily. In cervical cancer, intracavitary brachytherapy (ICBT) has been demonstrated to 
play a decisive role in curative radiation therapy (1). Even the most precise external beam radiation therapy (EBRT) like intensitymodulated radiation therapy (IMRT) and stereotactic radiation therapy cannot replace brachytherapy without lowering progression-free and overall survivals (2). ICBT of cervical cancer uses tandem and ovoid applicators. In ICBT, the dose has been prescribed traditionally to an imaginary point $\mathrm{A}$ in the paracervical triangle according to the Manchester method (3). Orthogonal x-rays can locate the point A that lies $2 \mathrm{~cm}$ lateral to the intrauterine tandem at the point $2 \mathrm{~cm}$ cranial from the external os. Point $\mathrm{A}$ is anatomically corresponding to the point where the uterine artery crosses the ipsilateral ureter. However, precise anatomical relationships between the tumor, the organs at risk (OARs), and point $\mathrm{A}$ is not obvious with orthogonal $\mathrm{X}$-rays.

Three-dimensional image-guided brachytherapy (3D-IGBT) has been applied in clinical practice since around 2000, where CT or MRI of the treated regions is performed to clarify the $3 \mathrm{D}$ anatomy of the gross tumor volume (GTV) and the OARs using CT/MRI compatible applicators in the treatment position (4). With the introduction of 3D-IGBT, it has become clear that a large tumor often does overgrow point $\mathrm{A}$, and brachytherapy dose prescribed to point $A$ is not enough to encompass the large tumor $(5,6)$. Some tumors recur in the low-dose region lying out of point A. MRI has been shown to play a decisive role in 3D-IGBT of cervical cancer because of an excellent soft-tissue contrast for delineation of anatomical structures $(1,4,7)$. According to the EMBRACE studies, high-risk clinical target volume (HR-CTV) of brachytherapy is defined as the residual tumor just before ICBT plus the cervix that are revealed by T2 weighted images (T2WI) of MRI, and ICBT should deliver boost dose to this HR-CTV up to more than $85 \mathrm{~Gy}$ in equivalent dose assumed to be delivered by $2 \mathrm{~Gy}$ fractions $\left(\mathrm{EQD}_{2}\right)$ including the dose from $\operatorname{EBRT}(4,5,8)$. Therefore, MRI with the applicators in situ is used as a gold standard in the EMBRACE studies. In contrast, 3D-IGBT using CT has a problem of difficulty in differentiating soft tissue contrasts and HR-CTV extracted by CT with the applicators in situ is usually larger than the HR-CTV delineated by T2WI (9). However, many institutions have difficulties in using MRI for each brachytherapy session due to logistic issues and the situation is especially serious in Japan, where less than $3 \%$ of the institutions performing HDR-ICBT are routinely using MRI for each ICBT session (10). CT is a much-preferred modality to be used in radiation oncology in many countries other than in Europe. Studies have shown that with a help of diagnostic MRI without the applicators in situ, HR-CTV can be contoured consistently by CT, and consensus guidelines for CT delineation of HR-CTV at the time of brachytherapy have been published $(9,11)$. Additionally, meta-analyses have disclosed the superiority of 3DIGBT compared with two-dimensional brachytherapy planning with point $\mathrm{A}$ in local control and morbidities $(12,13)$.

\section{HYBRID BRACHYTHERAPY OF THE CERVICAL CANCER}

With the EMBRACE studies, the long-standing controversy of radiation oncology was solved whether a boost radiation dose should be applied to the initially involved tumor volume or the shrunken residual tumor volume. Boost dose is now administered to the residual tumor volume, represented by HR-CTV. It is possible to raise the dose to the large residual cervical tumor by increasing dwell times of the ipsilateral ovoid applicator in ICBT, despite the inevitable increments of rectal and bladder doses. As only 3 applicators are used in ICBT, the possibility of dose distribution modification is very limited. If interstitial applicators are inserted into the parametrial region lateral to point $\mathrm{A}$ in addition to the intracavitary applicators, a rise of tumor dose is possible without increasing the rectal and bladder doses (Figure 1) $(4,5,14)$. Yoshida et al. have demonstrated by a simulation study that the tumor larger than $4 \mathrm{~cm} \times 3 \mathrm{~cm} \times 3 \mathrm{~cm}$ had better be treated by a combination of ICBT and interstitial brachytherapy (ISBT) [hybrid brachytherapy (HBT)], not by ICBT alone (15). Both vaginal and perineal approaches are available for the insertion of interstitial applicators into the parametrium. Specialized vaginal applicators suitable for transvaginal application were developed $(16,17)$ and commercially available. However, in Japan, these specialized applicators are not available and many institutions are doing free-hand transvaginal and/or perineal interstitial applications. The interstitial applicators are implanted under transrectal ultrasound guidance (Figure 2), and they are inserted up to the cranial margin of the parametrial tumor. The transvaginal application causes post-removal bleeding, although infrequently, therefore gauze pressure packing of the vaginal cavity is mandatory after the transvaginal application. The transperineal application can cover even the posterolateral tumor extension along the uterosacral ligament easily, which is difficult by transvaginal application (Figure 3).

\section{CLINICAL TRIAL OF HYBRID BRACHYTHERAPY IN JAPAN}

While HBT appears to be superior to the ICBT alone in a large cervical cancer from the standpoint of dose distribution and local control (5), it remains to be studied whether HBT can be performed safely on a multi-institutional basis in Japan. In 2015, we launched the multi-institutional phase I/II clinical trial of HBT for locally advanced cervical cancer (18). Twenty leading institutions in Japan performing HDR brachytherapy of cervical cancer participated in the trial. The locally advanced cervical cancer with an initial diameter of more than $5 \mathrm{~cm}$ with T2WI was recruited as the first step (Figure 4). Patients with distant metastases were excluded. The registered patients underwent EBRT to the whole pelvis in a conventional fractionation with concurrent weekly cis-diaminedichloroplatinum (CDDP) of $40 \mathrm{mg} / \mathrm{m}^{2}$. If T2WI post-30 Gy EBRT disclosed a residual tumor larger than $4 \mathrm{~cm}$, the patients were entered in the final registration. The finally registered patients were treated by EBRT to the bilateral pelvic sidewalls with a central pelvic shielding up to $50 \mathrm{~Gy}$ including the whole pelvis dose. In many Japanese institutions, central shielding is used routinely for IIIB patients after 30 Gy of the whole pelvis EBRT (19), therefore central shielding pelvic radiation therapy was employed as the EBRT in this study, and IMRT was not accepted. 

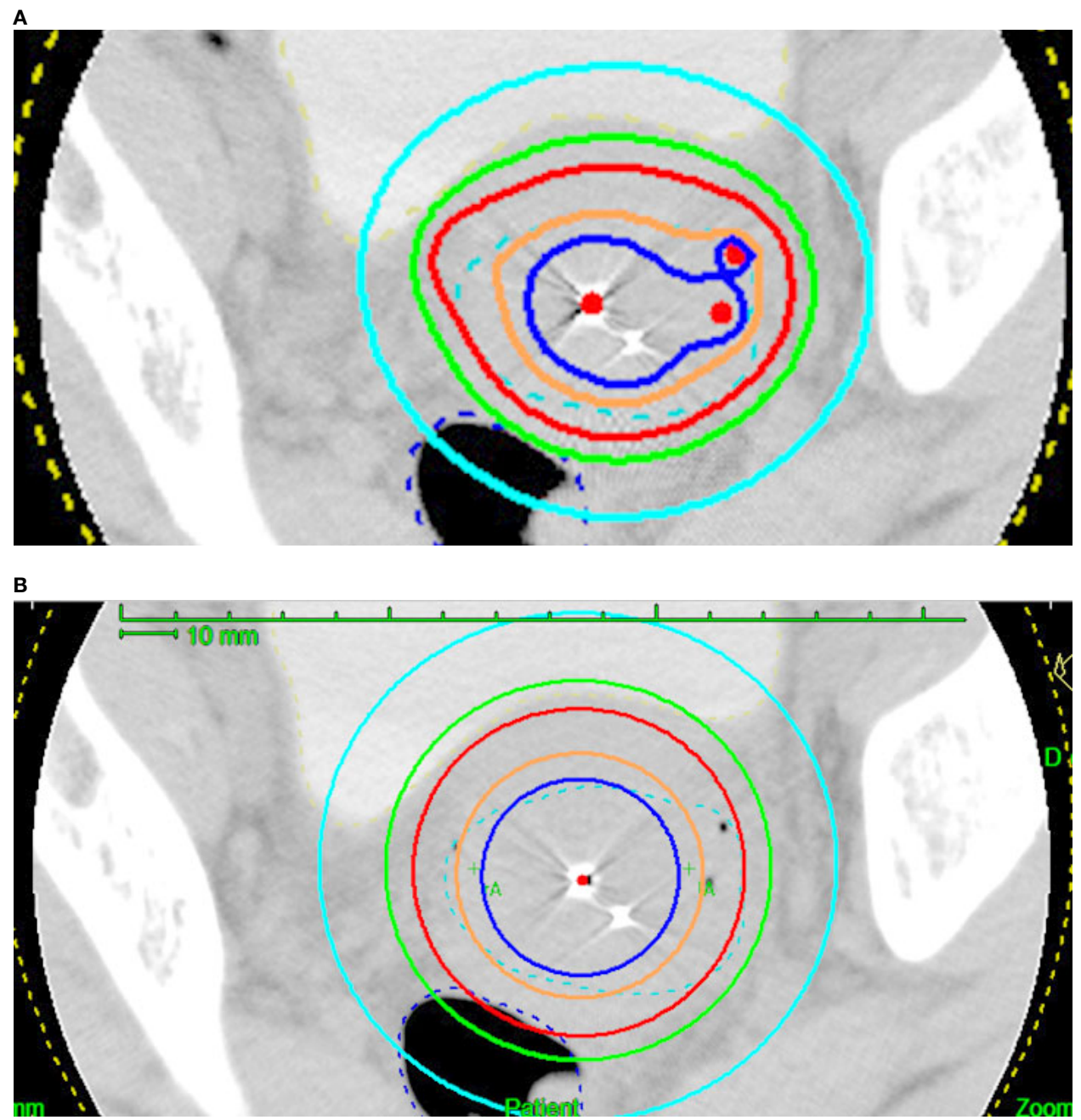

c

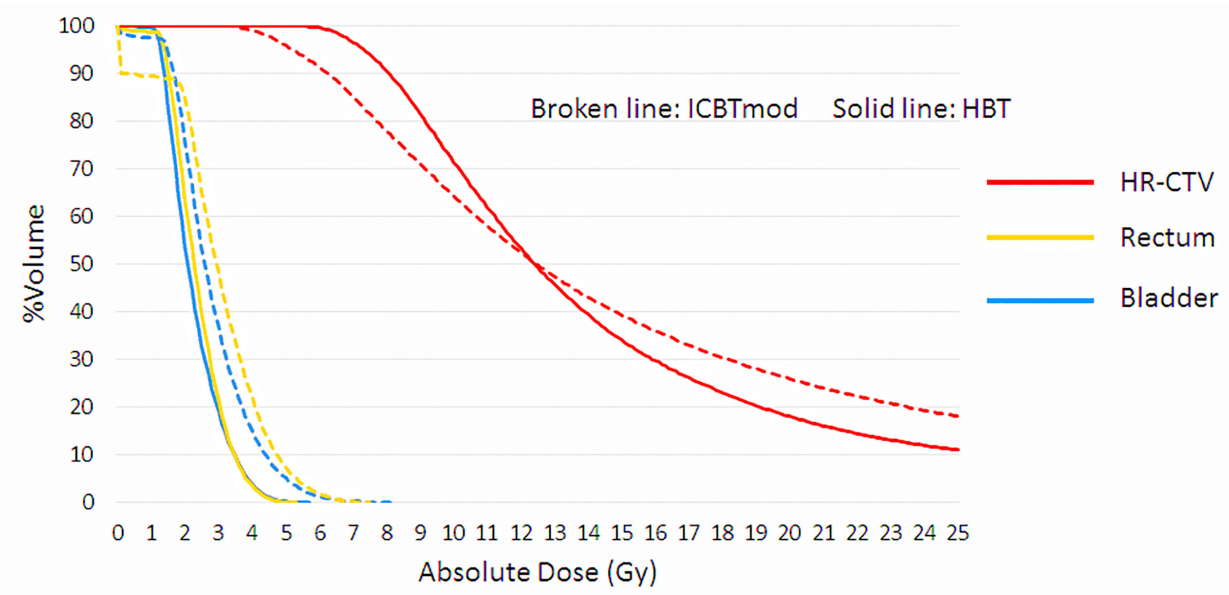

FIGURE 1 | (A) Dose distribution of hybrid brachytherapy (HBT) and (B) dose distribution of intracavitary irradiation with increasing dwell times of ovoid applicator (ICBTmod) to cover high-risk CTV with D90 $\geq 6$ Gy. (C) Dose-volume histogram (DVH) shows increased rectal and bladder doses in ICBTmod. HBT can deliver an adequate high-risk CTV dose without increment of rectal and bladder dose. 

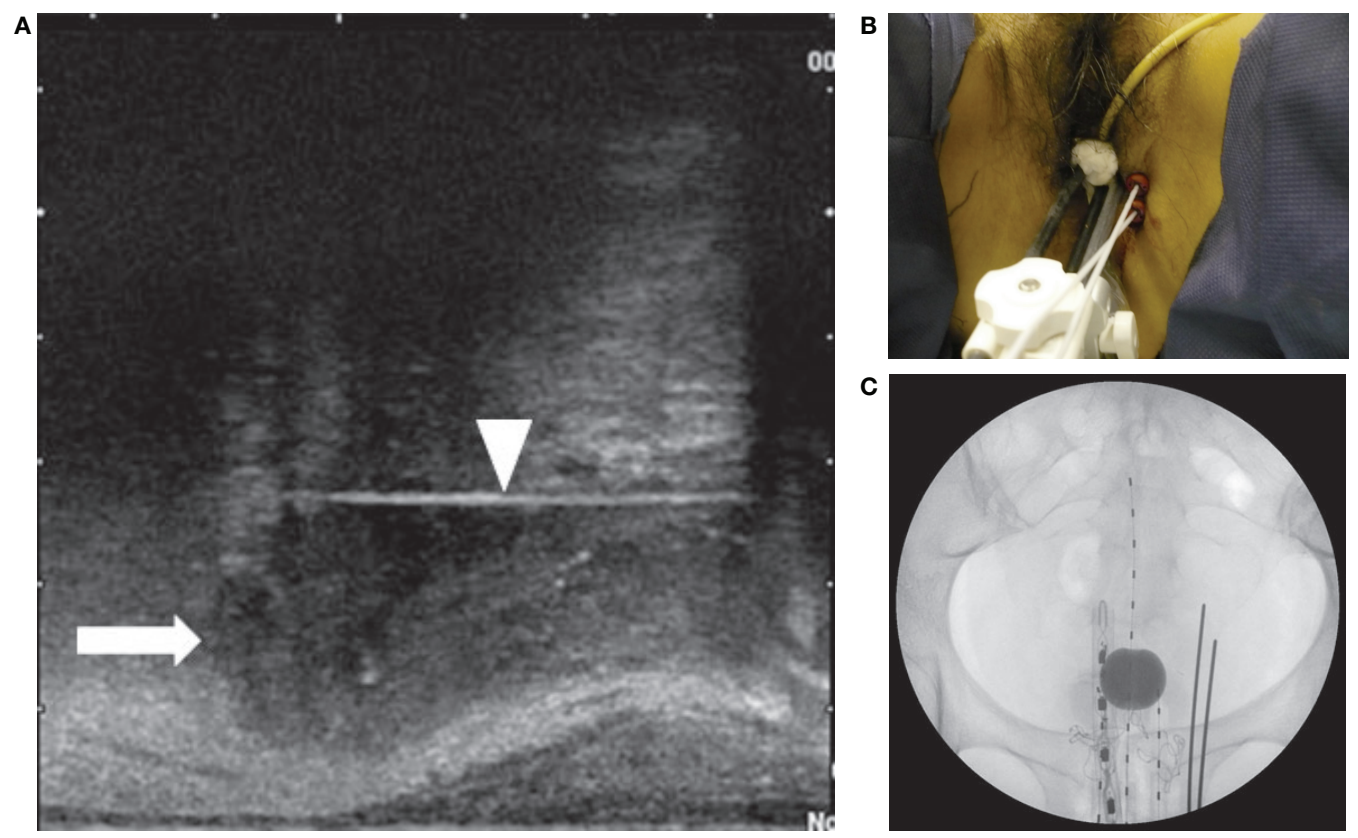

FIGURE 2 | (A) Sagittal view of the transrectal ultrasound showing the parametrial invasion (arrow) and the interstitial perineal applicator (arrowhead). The applicator is inserted up to the cranial margin of the parametrial invasion. (B) Two perineal interstitial applicators were inserted to the left of intracavitary applicators. (C) Anterior $\mathrm{X}$-ray of intracavitary and interstitial applicators. Also seen is a rectal dosimeter.

In Japan, currently, an increasing number of institutions are transitioning to IMRT from central shielding in order to increase the dose to the primary (20). HBT was administered with 6 Gy 4 times, twice weekly immediately after completion of the whole pelvic radiation therapy. The dose was prescribed to the HRCTV with $\mathrm{D} 90 \geq 6 \mathrm{~Gy} /$ fraction. Therefore, if the tumor dose delivered by the centrally shielded pelvic radiation is ignored, the tumor $\mathrm{EQD}_{2}$ is only $62 \mathrm{~Gy}$ in contrast to the EMBRACE recommendations of more than $85 \mathrm{~Gy}$ in $\mathrm{EQD}_{2}$ (7). On the day of HBT, EBRT was not performed. A number of the interstitial applicators should be less than 7 with a hyperdose sleeve (21) around the interstitial applicator less than $1 \mathrm{~cm}$ in

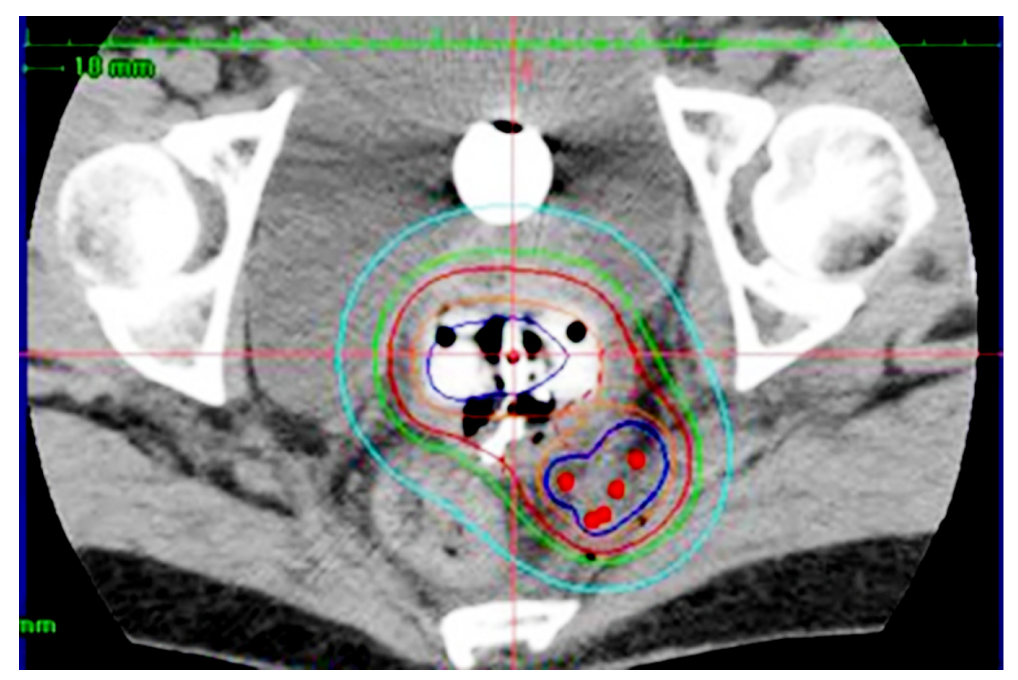

FIGURE 3 | Hybrid brachytherapy of cervical cancer extending along the left uterosacral ligament. Five red dots posterolateral to the intracavitary applicators are perineal interstitial applicators, with which good coverage of the uterosacral invasion is attained. 


\section{\begin{tabular}{|c|}
\hline $\begin{array}{c}\text { Uterine cervical cancer } \\
\text { PS 0-2, Age 20-75 } \\
\text { Tumor width larger than } 5 \mathrm{~cm} \\
\text { FIGO IB2, IIA2, IIB, IIIA, IIIB }\end{array}$ \\
Whole pelvic radiotherapy 30-30.6 Gy/15-17 f
\end{tabular}}

Tumor width larger than $4 \mathrm{~cm}$ assessed by MRI taken within one week before first brachytherapy

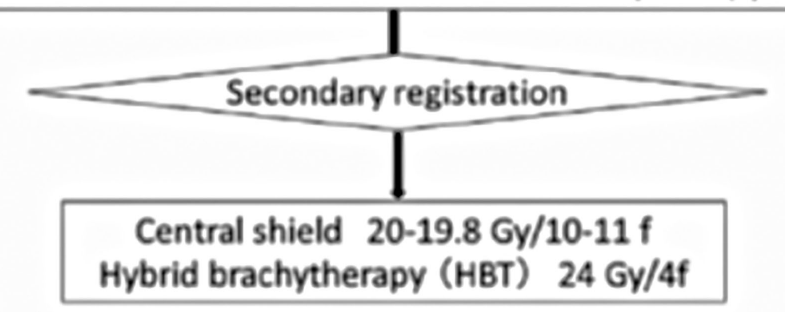

Tumor width smaller than $4 \mathrm{~cm}$ assessed by MRI taken within one week before first brachytherapy

FIGURE 4 | Shema of the phase I/II trial of hybrid brachytherapy.

diameter. Other dose constraints of HBT + EBRT are shown in Table 1 (7). Both MRI and CT are allowed to conduct 3D-IGBT, and the definition of contouring of HR-CTV was defined independently for this study (18). All 19 institutions but one used CT for each HBT session. If the parametrial tumor cannot be encompassed by 6 needle applicators with keeping hyperdose sleeves around the applicators of less than $1 \mathrm{~cm}$, the patients were treated by extensive ISBT using more than 6 needles and excluded from the study. If the residual tumor was smaller than or equal to $4 \mathrm{~cm}$ by T2WI after 30 Gy of EBRT and CDDP, they are treated by usual ICBT out of protocol. In the phase I portion, 20 patients were planned to be entered in the final registration and it was analyzed whether the HBT can be performed safely on a multi-institutional basis with less than $10 \%$ incidence of $\geq$ grade 3 acute nonhematological morbidities caused by HBT according to CTCAE ver. 4 (22). The detailed results will be published elsewhere, but till Feb. 2017, 20 patients were accrued to the phase I part of the study and only one patient had uterine bleeding from the transvaginal insertion site of grade 3 at the time of applicator removal. As the incidence of serious nonhematological morbidities was less than $10 \%(1 / 20,5 \%)$, the study progressed to phase II part to see the efficacy of HBT. Till the study closure in Oct. 2019, 73 patients were recruited for the 1 st registration and 52 patients (71\% of the 1 st registration) advanced to the final registration. Clinical characteristics of the patients are shown in Table 2. Although we had assumed that $50 \%$ of the 1 st-step registrants progressed to the final step, tumor shrinkage after $30 \mathrm{~Gy}$ of EBRT and weekly CDDP was not so remarkable as assumed and even $71 \%$ of the patients went to the final step and underwent HBT. As for dose constraints of the rectum, bladder, and sigmoid, 98.1\%, 100\%, and 100\% of the patients were irradiated per protocol, respectively. Quality of HBT was determined by HR-CTV D90 with $94.2 \%$ of the patient irradiated per protocol with a fractional HBT dose $\geq 6 \mathrm{~Gy}$. From the phase I portion, we could conclude that HBT can be performed safely and with a satisfactory quality of radiation

TABLE 1 | Dose constraints of HBT plus EBRT.

\begin{tabular}{|c|c|c|}
\hline & Per protocol & Acceptable deviation \\
\hline Rectum $D_{2}$ cc (Gy, $E Q D_{2}$, total dose) & $\leq 75$ Gy & Not applicable \\
\hline Bladder $\mathrm{D}_{2 \text { cc }}\left(\mathrm{Gy}, \mathrm{EQD}_{2}\right.$, total dose) & $\leq 90 \mathrm{~Gy}$ & Not applicable \\
\hline Sigmoid colon $\mathrm{D}_{2 \mathrm{cc}}\left(\mathrm{Gy}, \mathrm{EQD}_{2}\right.$, total dose) & $\leq 75$ Gy & Not applicable \\
\hline HR-CTVDgo (Gy, physical dose, per each HBT) & $\geq 6$ Gy & $\geq 5.4$ Gy \\
\hline Mean diameter of hyperdose sleeve around interstitial applicators (cm) & $\leq 1 \mathrm{~cm}$ & $\leq 1.5 \mathrm{~cm}$ \\
\hline
\end{tabular}

EBRT, external beam radiation therapy; $E Q D_{2}$, equivalent dose in 2 Gy fraction; HBT, hybrid brachytherapy; HR-CTV, high-risk clinical target volume. 
TABLE 2 | Characteristics of the patients recruited to the phase I/II trial.

\begin{tabular}{|c|c|c|}
\hline & $\begin{array}{l}\text { Primary registrants } \\
\qquad(n=73)\end{array}$ & $\begin{array}{l}\text { Secondary registrants } \\
\qquad(n=52)\end{array}$ \\
\hline \multicolumn{3}{|l|}{ PS } \\
\hline 0 & 52 & 37 \\
\hline 1 & 21 & 15 \\
\hline 2 & 0 & 0 \\
\hline \multicolumn{3}{|l|}{ Age } \\
\hline Median (years, range) & $48(26-74)$ & $48(26-74)$ \\
\hline \multicolumn{3}{|l|}{ FIGO Stage (2008) } \\
\hline IB2 & 11 & 10 \\
\hline$\| \mathrm{A} 2$ & 4 & 2 \\
\hline IIB & 29 & 20 \\
\hline IIIA & 1 & 0 \\
\hline \multirow[t]{2}{*}{ IIIB } & 28 & 20 \\
\hline & $\begin{array}{c}\text { Primary registrants } \\
\left(n=71^{\mathrm{a}}\right)\end{array}$ & $\begin{array}{l}\text { Secondary registrants } \\
\qquad(n=52)\end{array}$ \\
\hline \multicolumn{3}{|c|}{ Tumor width before treatment } \\
\hline Median (cm, range) & $5.70(4.30-9.20)$ & $5.70(4.30-9.20)$ \\
\hline \multicolumn{3}{|c|}{ Pelvic lymph node metastasis } \\
\hline No & 36 & 29 \\
\hline Yes & 35 & 23 \\
\hline \multicolumn{3}{|l|}{ Histopathology } \\
\hline SCC & 64 & 47 \\
\hline Adeno & 6 & 4 \\
\hline Adenosquamous & 1 & 1 \\
\hline Others & 0 & 0 \\
\hline
\end{tabular}

${ }^{a}$ Two patients retracted consent and their details are unknown.

Adeno, adenocarcinoma; Adenosquamous, adenosquamous carcinoma; FIGO, International Federation of Gynecology and Obstetrics; PS, performance status; SCC, squamous cell carcinoma.

delivery on a multi-institutional basis in Japan. The primary endpoint of the study was 2-year intrapelvic control and the final results of this phase I/II trial will be published soon elsewhere.

\section{INCREASED USE OF HYBRID BRACHYTHERAPY IN THE CERVICAL CANCER IN JAPAN}

The number of patients undergoing HBT is rising rapidly in Japan. According to the MicroSelectron Research Group in Japan (personal communication) which covers more than $80 \%$ of radiation facilities using HDR remote afterloading devices in Japan, a number of patients with cervical cancer undergoing HBT were 120 patients in 2015, 302 in 2017, and 349 in 2019 (Figure 5). The ratio of HBT in brachytherapy of cervical cancer increases correspondingly from $4.8 \%$ in 2015 to $13 \%$ in 2019. Many radiation oncologists are now accustomed to HBT procedure which is more invasive than ICBT, and they are now implementing HBT to increase the dose to the involved parametria. Educational seminars held by the specialists in HBT and by the vendor as well as site-visiting training by coaching doctors of the HBT clinical trial group contributed to the spread of HBT in Japan as well. Many Japanese radiation oncologists now realize that HBT can be easily performed and can deliver an adequate dose to the large parametrial invasion without increasing the rectal and bladder doses.

\section{CONCLUSIONS}

There is now abundant evidence that increased local control can be obtained with a larger dose in many cancer types $(5,14,23$, 24) and the HBT can deliver increased dose to cervical cancer without increasing doses to the OARs. An increasing number of institutions in Japan are now implementing HBT in the

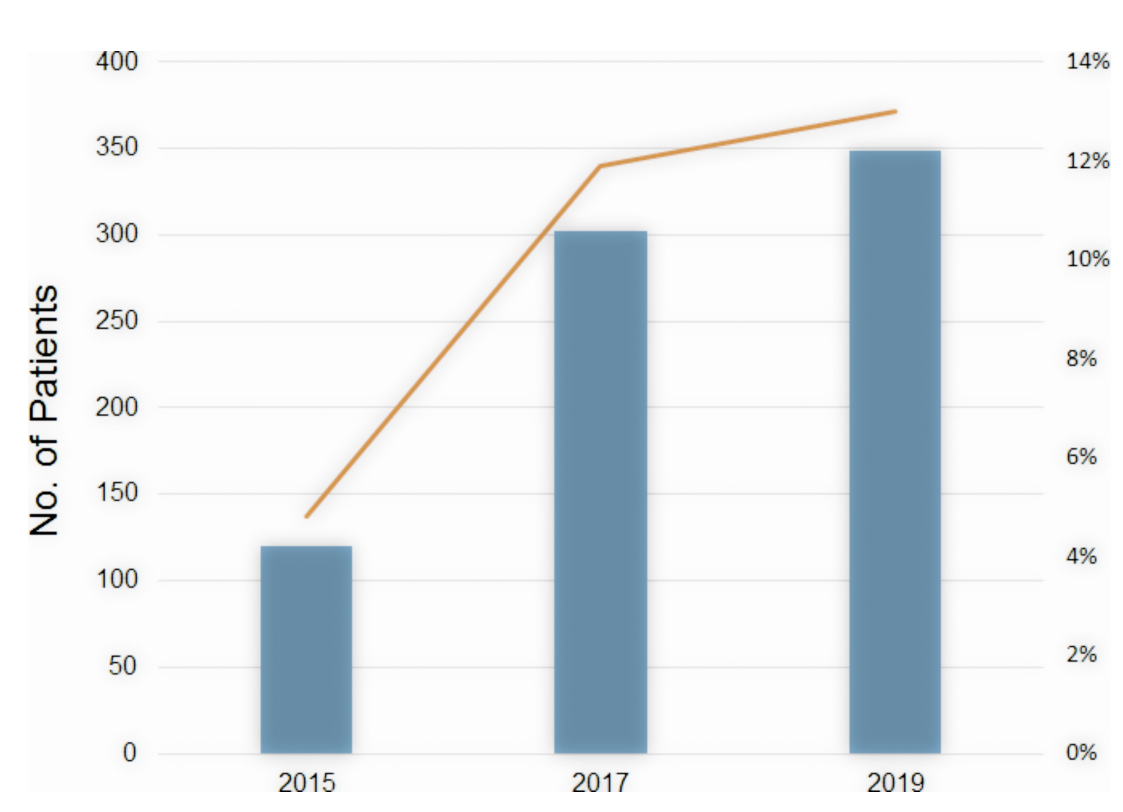

FIGURE 5 | The number of patients undergoing HBT in Japan (bar) and the ratio of HBT in brachytherapy of cervical cancer (line) by year. 
radiotherapy of locally advanced cervical cancer thanks to educational efforts. For the development of brachytherapy including HBT, hand-on education is mandatory. Furthermore, a multi-institutional randomized trial comparing ICBT vs. HBT is desired to establish the role of $\mathrm{HBT}$ in the radiotherapeutic management of cervical cancer.

\section{AUTHOR CONTRIBUTIONS}

Clinical trial planned by JI, NM, KO, and HO. MW, SS, TK, SK, $\mathrm{HH}, \mathrm{HIk}, \mathrm{KA}$, TO and HIg were responsible investigators of the participating institutes.

\section{REFERENCES}

1. Han K, Milosevic M, Fyles A, Pintilie M, Viswanathan AN. Trends in the Utilization of Brachytherapy in Cervical Cancer in the United States. Int J Radiat Oncol Biol Phys (2013) 87:111-9. doi: 10.1016/j.ijrobp.2013.05.033

2. Georg D, Kirisits C, Hillbrand M, Dimopoulos J, Potter R. Image-Guided Radiotherapy for Cervix Cancer: High-Tech External Beam Therapy Versus High-Tech Brachytherapy. Int J Radiat Oncol Biol Phys (2008) 71:1272-8. doi: 10.1016/j.ijrobp.2008.03.032

3. Tod MC, Meredith WJ. A Dosage System for Use in the Treatment of Cancer of the Uterine Cervix. Br J Radiol (1938) 11:809-24. doi: 10.1259/0007-128511-132-809

4. Pötter R, Dimopoulos J, Georg P, Lang S, Waldhausl C, Wachter-Gerstner N, et al. Clinical Impact of MRI Assisted Dose Volume Adaptation and Dose Escalation in Brachytherapy of Locally Advanced Cervix Cancer. Radiother Oncol (2007) 83:148-55. doi: 10.1016/j.radonc.2007.04.012

5. Pötter R, Tanderup K, Kirisits C, de Leeuw A, Kirchheiner K, Nout R, et al. The EMBRACE II Study: The Outcome and Prospect of Two Decades of Evolution Within the GEC-ESTRO GYN Working Group and the EMBRACE Studies. Clin Transl Radiat Oncol (2018) 9:48-60. doi: 10.1016/j.ctro. 2018.01.001

6. Murakami N, Kasamatsu T, Wakita A, Nakamura S, Okamoto H, Inaba K, et al. CT Based Three Dimensional Dose-Volume Evaluations for High-Dose Rate Intracavitary Brachytherapy for Cervical Cancer. BMC Cancer (2014) 14:447. doi: 10.1186/1471-2407-14-447

7. Pötter R, Haie-Meder C, Van Limbergen E, Barillot I, De Brabandere M, Dimopoulos J, et al. Recommendations From Gynaecological (GYN) GEC ESTRO Working Group (II): Concepts and Terms in 3D Image-Based Treatment Planning in Cervix Cancer Brachytherapy-3D Dose Volume Parameters and Aspects of 3D Image-Based Anatomy, Radiation Physics, Radiobiology. Radiother Oncol (2006) 78:67-77. doi: 10.1016/j.radonc.2005.11.014

8. ICRU. Prescribing, Recording, and Reporting Brachytherapy for Cancer of the Cervix: Report 89. J ICRU (2013) 13:1-258. doi: 10.1093/jicru_ndw027

9. Viswanathan AN, Erickson B, Gaffney DK, Beriwal S, Bhatia SK, Lee Burnett O3rd, et al. Comparison and Consensus Guidelines for Delineation of Clinical Target Volume for CT- and MR-Based Brachytherapy in Locally Advanced Cervical Cancer. Int J Radiat Oncol Biol Phys (2014) 90:320-8. doi: 10.1016/ j.ijrobp.2014.06.005

10. Toita T, Ohno T, Ikushima H, Nishimura T, Uno T, Ogawa K, et al. National Survey of Intracavitary Brachytherapy for Intact Uterine Cervical Cancer in Japan. J Radiat Res (2018) 59:469-76. doi: 10.1093/jrr/rry035

11. Mahantshetty U, Pötter R, Beriwal S, Grover S, Lavanya G, Rai B, et al. IBSGEC ESTRO-ABS Recommendations for CT Based Contouring in Image Guided Adaptive Brachytherapy for Cervical Cancer. Radiother Oncol (2021) 160:273-84. doi: 10.1016/j.radonc.2021.05.010

12. Kim YJ, Kang HC, Kim YS. Impact of Intracavitary Brachytherapy Technique (2D Versus 3D) on Outcomes of Cervical Cancer: A Systematic Review and Meta-Analysis. Strahlenther Onkol (2020) 196:973-82. doi: 10.1007/s00066020-01658-0

\section{FUNDING}

This work was supported by MHLW Research for Promotion of Cancer Control Programmes Grant Number 21EA1010.

\section{ACKNOWLEDGMENTS}

The authors gratefully acknowledge the generous clinical, research, and secretarial assistance of Dr. Koji Inaba, Dr. Kana Takahashi, Dr. Tairo Kashihara, Dr. Tomoya Kaneda, Dr. Yuko Nakayama, Dr. Ayaka Takahashi, and Dr. Yuri Shimizu of the Department of Radiation Oncology, National Cancer Center Hospital.

13. Suzumura EA, Gama LM, Jahn B, Campolina AG, Carvalho HA, de Soarez PC. Effects of 3D Image-Guided Brachytherapy Compared to 2D Conventional Brachytherapy on Clinical Outcomes in Patients With Cervical Cancer: A Systematic Review and Meta-Analyses. Brachytherapy (2021) 20:710-37. doi: 10.1016/j.brachy.2021.03.004

14. Pötter R, Tanderup K, Schmid MP, Jürgenliemk-Schulz I, Haie-Meder C, Fokdal LU, et al. MRI-Guided Adaptive Brachytherapy in Locally Advanced Cervical Cancer (EMBRACE-I): A Multicentre Prospective Cohort Study. Lancet Oncol (2021) 22:538-47. doi: 10.1016/S1470-2045(20)30753-1

15. Yoshida K, Yamazaki H, Kotsuma T, Takenaka T, Ueda MM, Miyake S, et al. Simulation Analysis of Optimized Brachytherapy for Uterine Cervical Cancer: Can We Select the Best Brachytherapy Modality Depending on Tumor Size? Brachytherapy (2016) 15:57-64. doi: 10.1016/j.brachy.2015.10.002

16. Dimopoulos JC, Kirisits C, Petric P, Georg P, Lang S, Berger D, et al. The Vienna Applicator for Combined Intracavitary and Interstitial Brachytherapy of Cervical Cancer: Clinical Feasibility and Preliminary Results. Int J Radiat Oncol Biol Phys (2006) 66:83-90. doi: 10.1016/j.ijrobp.2006.04.041

17. Nomden CN, de Leeuw AA, Moerland MA, Roesink JM, Tersteeg RJ, Jurgenliemk-Schulz IM. Clinical Use of the Utrecht Applicator for Combined Intracavitary/Interstitial Brachytherapy Treatment in Locally Advanced Cervical Cancer. Int J Radiat Oncol Biol Phys (2012) 82:1424-30. doi: 10.1016/j.ijrobp.2011.04.044

18. Murakami N, Kato S, Nakano T, Uno T, Yamanaka T, Sakurai H, et al. A Phase I/II Clinical Trial for the Hybrid of Intracavitary and Interstitial Brachytherapy for Locally Advanced Cervical Cancer. BMC Cancer (2016) 16:640. doi: 10.1186/s12885-016-2543-3

19. Tamaki T, Ohno T, Noda SE, Kato S, Nakano T. Filling the Gap in Central Shielding: Three-Dimensional Analysis of the EQD2 Dose in Radiotherapy for Cervical Cancer With the Central Shielding Technique. J Radiat Res (2015) 56:804-10. doi: 10.1093/jrr/rrv029

20. Hirai R, Tamaki T, Igari M, Kumazaki YU, Noda SE, Kato S. PlanOptimization Method for Central-Shielding Pelvic Volumetric-Modulated Arc Therapy for Cervical Cancer. Vivo (2020) 34:3611-8. doi: 10.21873/ invivo. 12206

21. Marinello BPG, Grimard L, Barret C. Dosimetry of Intraluminal Brachytherapy. Radiother Oncol (1992) 23:213-6. doi: 10.1016/S0167-8140 (92)80142-6

22. Common Terminology Criteria for Adverse Events (CTCAE) Version 4.0. US Department of Health and Human Services, National Institutes of Health, National Cancer Institute (2009).

23. Peeters ST, Heemsbergen WD, Koper PC, van Putten WL, Slot A, Dielwart MF, et al. Dose-Response in Radiotherapy for Localized Prostate Cancer: Results of the Dutch Multicenter Randomized Phase III Trial Comparing 68 Gy of Radiotherapy With 78 Gy. J Clin Oncol (2006) 24:1990-6. doi: 10.1200/ JCO.2005.05.2530

24. Onishi H, Shirato H, Nagata Y, Hiraoka M, Fujino M, Gomi K, et al. Stereotactic Body Radiotherapy (SBRT) for Operable Stage I Non-SmallCell Lung Cancer: Can SBRT be Comparable to Surgery? Int J Radiat Oncol Biol Phys (2011) 81:1352-8. doi: 10.1016/j.ijrobp.2009.07.1751 
Conflict of Interest: The authors report research grant from Elekta and ITOCHU and consultation honoraria from HekaBio, Alpha-TAU, and Palette Science.

Publisher's Note: All claims expressed in this article are solely those of the authors and do not necessarily represent those of their affiliated organizations, or those of the publisher, the editors and the reviewers. Any product that may be evaluated in this article, or claim that may be made by its manufacturer, is not guaranteed or endorsed by the publisher.
Copyright (@) 2022 Itami, Murakami, Watanabe, Sekii, Kasamatsu, Kato, Hirowatari, Ikushima, Ando, Ohno, Okamoto, Okuma and Igaki. This is an open-access article distributed under the terms of the Creative Commons Attribution License (CC BY). The use, distribution or reproduction in other forums is permitted, provided the original author(s) and the copyright owner(s) are credited and that the original publication in this journal is cited, in accordance with accepted academic practice. No use, distribution or reproduction is permitted which does not comply with these terms. 